\title{
EDITORIAL
}

\section{Challenges in the Management of Common Tropical Diseases During COVID-19 Pandemic}

\author{
Vasanthi Pinto $^{1^{*}}$, Amila Jayasinghe ${ }^{2}$ \\ ${ }^{1}$ Professor of Anaesthesia \& Intensive Care, ${ }^{2}$ Senior Lecturer in Anaesthesia \& Intensive Care, \\ Department of Anaesthesia \& Intensive Care, Faculty of Medicine, University of Peradeniya, Sri Lanka
}

In the tropical and subtropical regions of the world, some tropical diseases, such as Dengue, leptospirosis, malaria, seasonal influenza (H1N1), chikungunya, tuberculosis, scrub typhus, are already endemic to the population. Conscientiousness and persistence of care is necessary not to neglect other endemic tropical diseases during the current pandemic. Amid the current pandemic, there can be two occurrences: co-infection and co-occurrence.

Co-occurrence can be defined as having parallel presentations as COVID-19; and the presence of tropical diseases simultaneously as COVID-19 in the same patient is termed co-infection. Coinfections are not new; we can find multiple shreds of evidence from the world. ${ }^{1,2,3}$

Both these situations possess a challenge.

These tropical diseases can have the same presentation as COVID-19, leading to clinical and laboratory diagnostic dilemmas of both conditions. Some tropical diseases can modify the clinical presentation of COVID-19 and result

*Correspondence: Vasanthi Pinto
E mail: vasantipinto@yahoo.com

https://orcid.org/0000-0001-6938-248x

DOI: https://doi.org/10.4038/slja.v29i2.8935

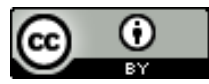

in difficulty in the diagnosis and treatment. In most tropical regions, clinicians are increasingly challenged to determine these febrile illnesses without diagnostics but clinical presentation. This bears the risk of these tropical diseases going under-reported.

The spectrum of COVID-19 spans from an acute febrile illness to severe respiratory problems. Most above diseases also complicate in the same manner.

\section{Dengue and COVID-19:}

WHO estimates there to be 100 million cases of Dengue (virus DENV) annually in Asia. In Sri Lanka, we have a high prevalence, with two peaks occurring with the monsoon rains in JuneJuly (higher) and October-December. In 2020, we had 31162 dengue cases, and from January 2021 up to now 10613 cases. During the second quarter of 2020 , there was a $73.6 \%$ reduction of Dengue compared with the same period in 2019. ${ }^{4}$ The period during which the impact of the first COVID-19 outbreak was at its highest, reported cases of Dengue were at their lowest. The decrease may be partly explained by the changes in rainfall patterns but cannot be only explained by weather conditions. Under-reporting of cases may be one explanation, with the possibility that Dengue patients may not have presented to hospitals due to fear of hospital-acquired COVID-19 infection. This poses a question, whether we have missed Dengue cases? This question has its own credits. The similar clinical 
manifestations shared by COVID-19 may have led to a diagnostic challenge, specifically, when patients develop haemoconcentration, thrombocytopenia, leukopenia, increased liver enzymes, erythematous skin rash, petechiae, and urticaria.

One example was Argentina in 2020. With an epidemic of Dengue, retrospectively identified hidden cases of SARS-CoV-2 was only $2.85 \%$ of samples tested. ${ }^{5}$ The danger could be, these undiagnosed missed cases may have contributed to the fast expansion of the SARS-CoV-2 outbreak in some countries. ${ }^{6,7}$

In Sri Lanka too, we have had patients initially confirmed of Dengue (IgM and $\mathrm{IgG}$ ) later found to be positive for Covid-19. Subsequent analysis failed to detect DENV, confirming the absence of dengue infection.

This misdiagnosis is probably due to serology cross-reactivity between COVID-19 and DENV antibodies. There are few hypotheses for crossreactivity; patients with previous exposure to DENV have anti-DENV antibodies that are crossreactive with SARS-CoV-2 antigens. In the same way, there may be antigenic similarities between SARS-CoV-2 and DENV, such that upon SARSCoV-2 infection, the body is triggered to generate anti-DENV antibodies derived from memory B cells.

The serological cross-reactivity between SARSCoV-2 and DENV might have caused the falsepositive phenomena in rapid dengue serology tests. Pre-existing DENV-antibodies might potentially affect COVID-19 through antibodydependent enhancement. This problem can have a different magnitude; the potential consequences of overlapping immunological cascades between Dengue and COVID-19 will affect disease severity and vaccine development. ${ }^{8}$

Sri Lanka frequently uses rapid antigen tests to diagnose COVID-19 but has limited sensitivity.
The need for specific tests to differentiate COVID-19 and Dengue can be highlighted.

There are many consequences of misdiagnosis. DHF and COVID-19 both share common features in pathogenesis, leading to a cytokine storm, affecting the integrity of the vascular endothelium leading to vasculopathy, coagulopathy and capillary leak. Both share thrombocytopenia; in Dengue, NS1 induces complement-mediated pathways, which cause platelet lysis, whereas SARS-CoV-2 inhibits bone marrow growth and induces apoptosis. The activity of coagulation factors, including prothrombin, factors V, VII, VIII, IX, and X, antithrombin, and $\alpha 2$-antiplasmin, are markedly reduced during Dengue. In contrast, these are high in the COVID-19 patients leading to a hypercoagulable state in which, pathobiology of coagulopathy is not well understood.

Therapeutic considerations could be many. Fluid therapy is one such diversity. In DHF, a substantial volume of fluid therapy is recommended. In contrast, fluid management has to be done cautiously with COVID-19 as COVID patients have ARDS and are more prone to develop pulmonary oedema. Aggressive fluid resuscitation is only recommended for COVID19 patients in shock, for initial resuscitation. Hence for either infection, close clinical monitoring of fluid status is required.

Another cutting-edge controversy is LMWH. LMWH is used for management of moderate to severe COVID-19 cases due to increased risk of thrombosis. In contrast, Dengue complicated with coagulopathy will need correction of thrombocytopenia and clotting deficits.

There is a possibility of change in the DENV serotype circulating during the COVID-19 pandemic in the region, which might lead to problems in future. 
There are many indirect effects of COVID-19 over Dengue. The vector control measures, and dengue prevention programs were reduced. Continuous dengue surveillance needs to be in place to track transmission dynamics of DENV infection in the region.

\section{COVID-19 Epidemic and Leptospirosis}

COVID-19 and leptospirosis are two entirely different infections but have many similarities, including the same incubation period, the pattern of respiratory involvement, and poor outcomes. Cytokine storm is considered the main incriminating factor in both, leading to pulmonary complications, immune-mediated vasculitis, increased permeability, and alveolar bleeding.

Sri Lanka has experienced significant and frequent outbreaks of leptospirosis during the last decade, and the mortality rates were higher than Dengue. ${ }^{9}$ The estimated annual incidence of leptospirosis in Sri Lanka was 300 per 100,000 people. More vigilance on COVID has a risk of inattention on leptospirosis, but in contrast to Dengue, an increase in the case number was seen. ${ }^{10,11}$ Increased farming activity that was actively encouraged by the government during the lockdown and the non-implementation of the state-sponsored doxycycline prophylaxis program for high-risk individuals could be the reasons.

There can be a diagnostic dilemma since no single diagnostic test for leptospirosis with high positive and negative predictive values is defined. In Asian countries with limited resources, we need affordable and accurate diagnostic methods to prevent misdiagnosis of leptospirosis, which require specific management. Antimicrobial therapy needs to be initiated as soon as the diagnosis and should be continued for an entire course, need of dialysis even in the presence of polyuria, plasma exchange, corticosteroids and intravenous IG also should be considered.

\section{Scrub Typhus; Rickettsia Infections}

In the recent past, scrub typhus outbreaks have also been reported in many parts of the Asia. In Sri Lanka, the dry regions of the country with jungles have a predominance of scrub typhus, while the mountainous areas have a predominance of spotted fever. Scrub typhus infection may co-exist with COVID-19. Both infections can cause a maculopapular rash, multiple organ involvement, vasculopathy, myocarditis, and progress to ARDS. Tests for a likely co-infection should be undertaken whenever suspected. Availability of rapid diagnostic kits for scrub typhus should be ensured in COVID treatment facilities.

\section{Hantavirus infections}

Hantaviruses can produce a disease, leading to a diagnostic dilemma, especially with Hantavirus Pulmonary Syndrome; a severe pulmonary illness with a high mortality rate of $38 \%$. The chest radiographs usually reveal marked peri-bronchial cuffing and Kerley B lines early in the disease. Rapid progression of bilateral interstitial and alveolar infiltrates are sometimes indistinguishable with COVID-19.

\section{Tuberculosis and COVID-19}

Either disease can occur in any order, but coexistence poses a challenge in the differential diagnosis. COVID-19 can appear during anti-TB treatment and limited or no protection against COVID-19 might have favoured SARS-CoV-2 infection.

The lack of studies on the differential diagnoses of COVID-19 in tropical areas makes the management of both the diseases extremely challenging. Also, it is understandable that this pandemic has repressed the healthcare systems, and all resources have been diverted to COVID 19, which needs reconsideration. 


\section{References}

1. Nguyen Than Ha Quyen et al. Am J Trop Med Hyg, 2017 Jul; 97(1):146-150.

2. Challenges Imposed on the diagnoses by Dengue, Zika, and, Chikungunya with same symptoms Paolo Marinho de Andrade Zanotto, 2018 Aug 28;9:1964.

3. A Roth et al. Concurrent outbreaks of Dengue, chikungunya, and Zika virus infections - an unprecedented epidemic wave of mosquito-borne viruses in the Pacific 2012-2014, 2014 Oct 16; 19(41)

4. Data from the Epidemiology Unit of the Ministry of Health in Sri Lanka

5. Lucila M Carosella et al. Characteristics of Patients Co-infected with Severe Acute Respiratory Syndrome Coronavirus 2 and Dengue Virus, Buenos Aires, Argentina, March-June 2020.

6. Lorenzo L S et al. Covert cases of SARSCoV-2: An obscure but present danger in regions endemic for Dengue and Chikungunya viruses. 2021 Jan 6; 16(1)
7. Mateus Santana do Rosário. Concerns about COVID-19 and arboviral concurrent outbreaks Braz J Infect Dis, Nov-Dec 2020; 24(6):583-584

8. Harapan $\mathrm{H}$ et al. Covid-19 and Dengue: Double punches for dengue-endemic countries in Asia. Med Virol, 2021 Mar; 31(2): e2161.

9. Warnasekara J, Koralegedara I, Agampodi S. Estimating the burden of Leptospirosis in Sri Lanka; a systematic review. BMC Infectious Diseases Volume 19,119 (2019)

10. Official websites for Sri Lanka's response to COVID-19. https://covid19.gov.lk. Epidemiology Unit Ministry of Health \& Indigenous Medical Services. Coronavirus disease 2019 (COVID-19) - Situation Report http://www.epid.gov.lk

11. Niriella M A et al., Dengue and leptospirosis infection during the coronavirus 2019 outbreak in Sri Lanka, Trans R Soc Trop Med Hyg. 2021 Apr. 